\title{
Local Changes of Medium in Studies of Individual Cells
}

\author{
Staffan Dryselius, Per-Eric Lund and Bo Hellman* \\ Department of Medical Cell Biology, Uppsala University, Biomedicum, Box 571, S-751 23 Uppsala, Sweden
}

Key words: superfusion/single cells/solution changes/dextran-linked rhodamine B/ ATP-regulated $\mathrm{K}^{+}$channels

\begin{abstract}
$A B S T R A C T$. A procedure is described for changing the medium surrounding individual cells attached to the bottom of a cell chamber. A small hole at the "apex" of a plastic U-tube allowed application and withdrawal of medium. The medium to be applied was perfused through the $U$-tube by pressure at one end and suction at the other. To prevent premature delivery of new medium from the U-tube, suction of the outlet dominated resulting in a net withdrawal of medium from the cell chamber. The flow of medium through the hole could be reversed rapidly by arresting the suction with an electromechanical valve. In this way it was possible to obtain $95 \%$ replacement of medium within $60 \mathrm{~ms}$. A pressure transient arising from the closure of the valve was damped by the presence of a small air bubble in the system. To secure a precise deposition of medium and minimize the risk of mechanical disturbances to the cell it was essential to be able to inspect the medium changes visually. For this purpose the fluorescent indicator rhodamine B bound to dextran proved satisfactory. Free rhodamine B could not be used because it had biological effects, as was evident from studying ATP-regulated $\mathrm{K}^{+}$channels in pancreatic $\beta$-cells. When using a purpose-designed syringe pump for perfusing the $U$-tube, the technique allows well controlled exposure of individual cells to test substances added together with dextran-linked rhodamine $B$.
\end{abstract}

With the introduction of the electrophysiological patch clamp technique (9) and the design of fluorescent probes enabling ratiometric recordings of ion activities $(4,8,10)$ there has been a growing interest in measurements on single cells. Studies of how different substances interact with single cells are critically dependent on methods for inducing well controlled and rapid changes of the extracellular environment $(1,2,5,11$, 12, 13, 16). Among the techniques proposed for rapid medium changes around individual cells, the U-tube approach shows certain promise $(5,12)$. In the present study it is described how a further development of this technique makes it possible to establish a well defined microenvironment with minimal disturbances to the cells.

\section{MATERIALS AND METHODS}

General features and operation of the medium exchange system. All parts in contact with media are constructed of chemically inert components. The essential parts of the system are outlined in Fig. 1. A U-shaped tube (panel A) was mounted on a micromanipulator to enable its precise positioning when submerged in a cell chamber. The U-tube was provided with a hole at the "apex" and held about $50 \mu \mathrm{m}$ from a cell, which had been allowed to attach to the bottom of the chamber during culture. The U-tube was filled with the desired medium

\footnotetext{
* Address for correspondence.
}

by positive pressure applied at one end. Sufficient suction was applied to the other end to create a net flow into the hole from the medium outside. This procedure effectively prevented outward diffusion of medium from the U-tube. By halting the suction with an electromechanical valve (No. 1201618H, Lee Co, Westbrook, CT, USA) the flow through the hole was reversed. The medium in the U-tube then rapidly replaced that surrounding the cell. This process could be reversed by reopening the valve, resulting in withdrawal of the medium previously applied. All medium changes were indicated together with other recorded data by letting the valve switchings trigger an electric pulse.

An air bubble in the system (Fig. 1) served as a damping device for diminishing recoil pressure transients generated by the electromechanical switching. The air bubble was enclosed in a $10 \mu \mathrm{l}$ chamber between the valve and the $200 \mathrm{~mm}$ long "suction arm" of the U-tube. The efficiency of damping depended on the volume of air in the chamber.

The combined positive pressure and suction applied to the U-tube was produced by a purpose-made slave syringe pump (Fig. 1, panel B) driven hydraulically by a commersial infusion pump (UNITA I, B. Braun Melsungen AG, Melsungen, Germany). A pair of glass syringes (Hamilton Nos. 1002 and 1005) with different bores were mounted opposite to each other in a push-pull system with their pistons attached to a common sliding block. Two hydraulic cylinders were symmetrically arranged around the joint axis of the syringes. These cylinders were fastened to the block, moving it along the axis of the syringes. The hydraulic cylinders were propelled by sili- 
con oil (MS 550, Dow Corning, Barry, UK) from the infusion pump. In this way, the syringe of a narrower bore was compressed at the same rate as the wider one was expanded, thus enabling a net suction of fluid through the hole in the U-tube. When the syringe pump was used for electrophysiological studies, noise was suppressed by placing the driving infusion pump outside the Faraday cage accomodating the rest of the equipment.

The sliding seals of the cylinders and syringes were made from low friction plastics. It is important to avoid materials which differ too much in friction coefficients between rest and motion. This counteracts discontinuous movement of the syringes due to stick-slip effects. Nitril rubber O-rings proved satisfactory for making seals against the brass hydraulic cylinders, and polytetrafluorethylene (PTFE) was used in the glass syringes. The function of the U-tube is sensitive to gas bubbles, which can disturb the flow pattern at the ejection hole. It was found that bubble formation could be essentially pre-

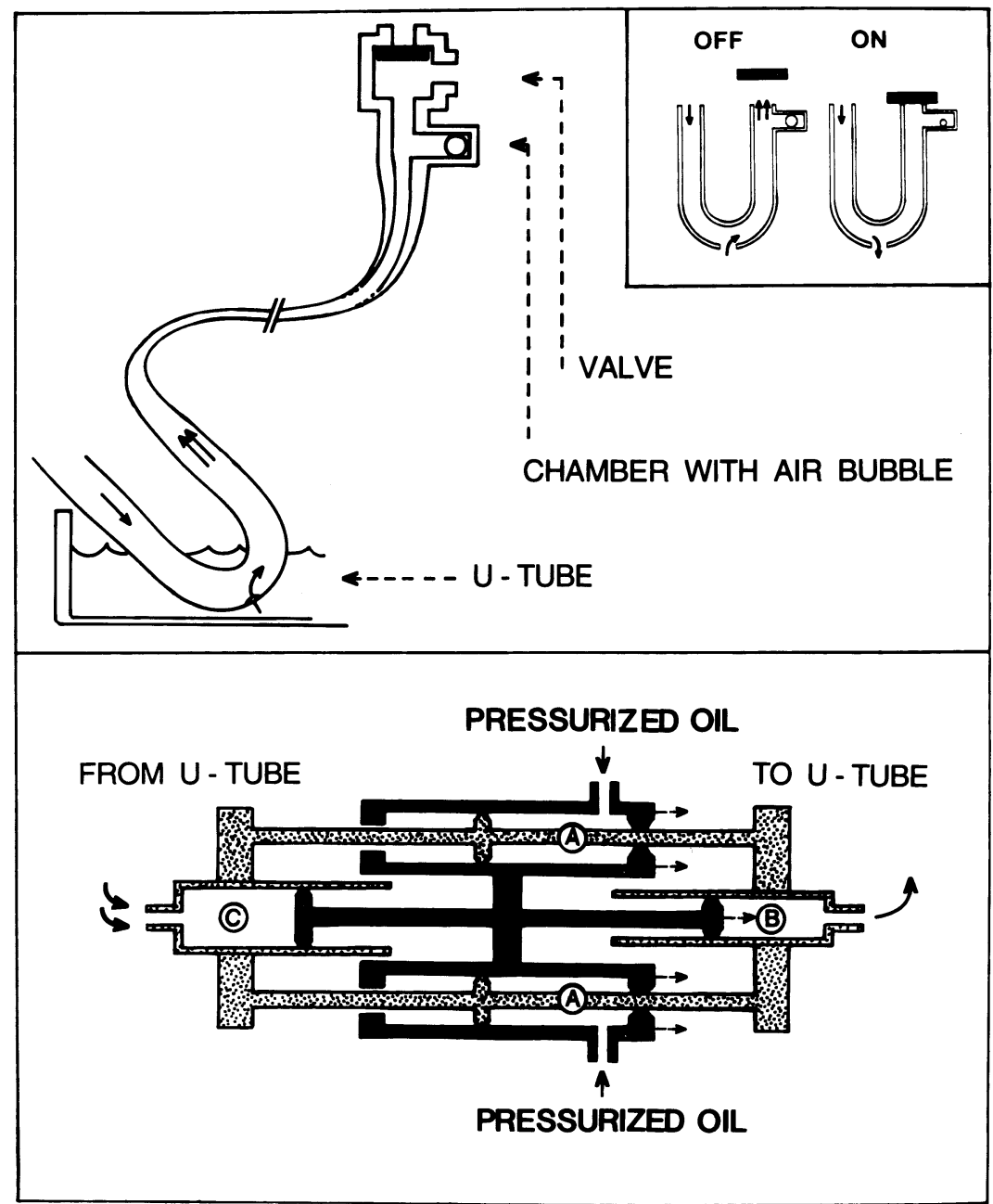

Fig. 1. Device for the rapid application of test substances to individual cells. A plastic tube (upper panel) with an inner diameter and wall thickness of $0.1 \mathrm{~mm}$ was bent into a U-shape. A small hole $(30 \mu \mathrm{m})$ was made at the "apex" of the tube. The U-tube was perfused with the medium to be applied by positive pressure at one end and suction at the other. The hole was positioned about $50 \mu \mathrm{m}$ from a cell, which had been allowed to attach to the bottom of a chamber. Initially, the inlet flow of medium to the U-tube was kept lower than the outflow, resulting in withdrawal of medium from the chamber. This effectively prevented premature exposure of the cell to the test substance. The relative rates of inflow and outflow of the U-tube and the flow through the hole are indicated by single or double arrows. The tube is connected to an electromechanical 3way valve at the outlet, enabling the suction to be directed to a reservoir instead of the U-tube. By switching the valve from "off" to "on" mode (upper panel, inset) the suction is arrested while the pressurized inflow is left unaffected, thus reversing the flow through the hole to allow new medium to reach the cell. Pressure transients induced by the switching are damped by the presence of an air bubble enclosed in a $10 \mu l$ chamber between the valve and the U-tube. A hydraulically driven syringe pump (lower panel) supplies the U-tube with both positive pressure and suction. An external driving unit injects hydraulic oil into the twin cylinders (A) of the pump, forcing the parts shown in black, including the pistons of the syringes (B and C), to slide relative to the stationary parts (dotted areas). Due to the push-pull arrangement and the narrow bore of syringe $\mathrm{B}$, fluid is expelled from this syringe $(1 \mu \mathrm{l} / \mathrm{min})$ at half the rate of suction into $\mathrm{C}(2 \mu \mathrm{l} / \mathrm{min})$. 
vented by making the arm of the U-tube connected to the outflow syringe shorter than $250 \mathrm{~mm}$.

Making the hole. The U-tube was made from PTFE tubing with an inner diameter and wall thickness of $0.1 \mathrm{~mm}$. When making the hole a length of tubing was sealed at one end and slightly pressurized by connecting the other end to a manually operated syringe filled with air. With the aid of a micromanipulator, the tube was then made to approach closely, but not to touch, a hot glass spike melted on to an electrically heated platinum filament. On approaching the hot spike, a depression formes in the wall of the tube. As the wall becomes softer, it bulges towards the spike whereby more material melts away until a $30 \mu \mathrm{m}$ opening is formed. As air begins to stream out, the spike and filament cool down rapidly, preventing further widening of the hole.

\section{RESULTS}

Time course of medium changes. The delivery of medium was inspected microscopically by introducing $1 \mathrm{mM}$ methylene blue in the U-tube. The time for completing medium changes was assessed by recording changes in pipette current essentially as described by

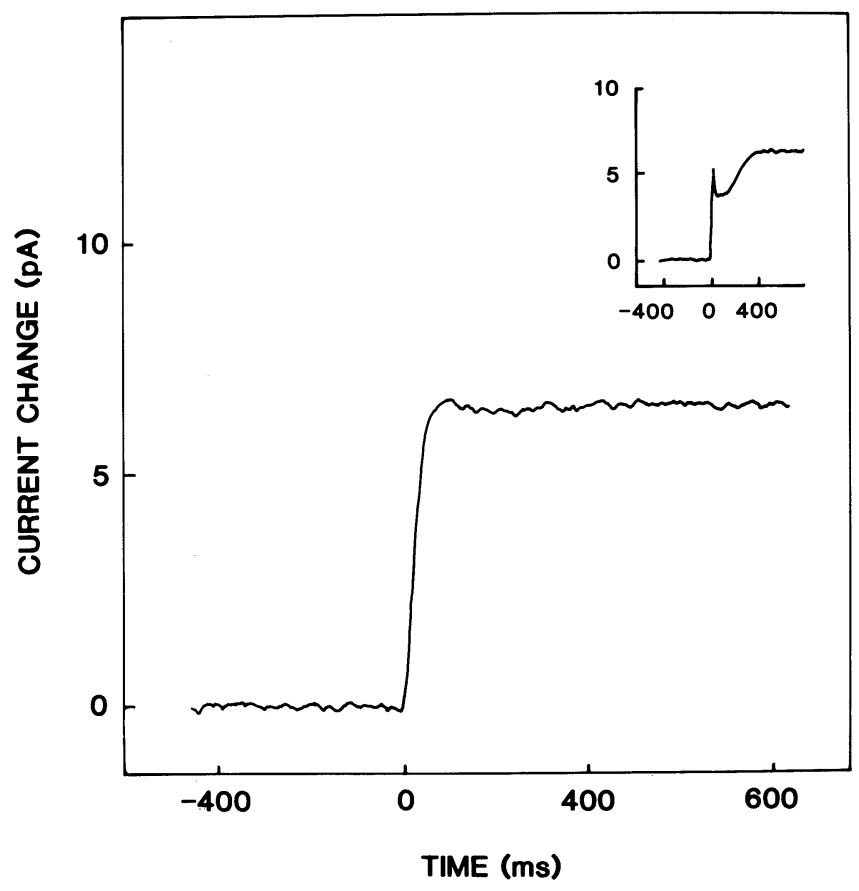

Fig. 2. Time course of a medium change measured close to the hole of a U-tube perfused with $150 \mathrm{mM} \mathrm{KCl}$ and with a flow through the hole of $1 \mu \mathrm{l} / \mathrm{min}$. The hole was positioned $50 \mu \mathrm{m}$ from the tip of an open $10 \mathrm{M} \Omega$ patch clamp pipette filled with $150 \mathrm{mM} \mathrm{NaCl}$ that was immersed in a medium of the same composition. At zero time the medium at the pipette tip was changed and a current shift was evoked by altered ionic conditions around the tip. The inset shows a situation where recoil pulses generated by switching the valve are left unsufficiently damped.
Brett et al. (2). Fig. 2 shows a medium change as a function of time, indicating $95 \%$ exchange in $60 \mathrm{~ms}$ at 50 $\mu \mathrm{m}$ distance and with the damping set for an overshoot of $5 \%$. When excluding the air bubble, microscopic inspection revealed a high velocity protuberance that produced a biphasic exchange pattern (Fig. 2, inset).

Reactions of living cells to medium changes. The activity of ATP-regulated $\mathrm{K}^{+}$channels was chosen as an indicator of metabolism. The measurements were made at $20-22{ }^{\circ} \mathrm{C}$ on individual pancreatic $\beta$-cells using the cell-attached configuration of the patch clamp technique (9) with the U-tube placed at a distance of $50 \mu \mathrm{m}$. The cells were prepared according to a previously described protocol and single channel $\mathrm{K}^{+}$currents were recorded with high $\mathrm{K}^{+}$in the pipette (7).

To achieve the desired changes of medium around a particular cell with minimal mechanical disturbances, it is essential to be able to follow the process microscopically. Methylene blue proved unsuitable, since concentrations of the indicator known to affect the cells were required $(14,15)$. When searching for other indicators to be used in lower concentrations, advantage was taken of the sensitivity of fluorescence detection. Excitation at long wavelengths was preferred in not requiring special equipment and in reducing photodamage to the cells (6). Among various alternatives Rhodamine B was tested in representing a widely employed fluorescent stain excitable at $570 \mathrm{~nm}$.

Visualisation of the medium changes required the presence of $5-10 \mu \mathrm{g} / \mathrm{ml}$ rhodamine B. These concentrations had themselves effects on pancreatic $\beta$-cells. In Fig. 3 it can be seen how the ATP-regulated $\mathrm{K}^{+}$channels were affected by rhodamine $\mathrm{B}$ in the presence of 3 $\mathrm{mM}$ glucose. The channel openings were temporarily replaced by action potentials and did not reappear until 30-60 s after the action potentials had decayed. In addition to channels with the characteristics of ATP-regulated ones, also larger channels appeared. In Fig. 4, panel A summarises the time dependent effects of 5-10 $\mu \mathrm{g} / \mathrm{ml}$ rhodamine B on the ATP-regulated $\mathrm{K}^{+}$channel activity of the pancreatic $\beta$-cells. Even when introduced at a concentration of $1 \mu \mathrm{g} / \mathrm{ml}$, rhodamine B suppressed channel activity (Fig. 4, panel B).

In the attempts to avoid interference with the cells, further testing was performed with rhodamine B covalently bound to an effectively impermeant dextran of $10 \mathrm{kDa}$. This dextran complex (Molecular Probes Inc., Eugene, OR, USA) contained $0.5-2$ rhodamine residues per dextran molecule. The concentration was adjusted to be equivalent to $10 \mu \mathrm{g} / \mathrm{ml}$ free rhodamine B. Sufficient fluorescence was thus obtained without any effects on the ATP-regulated $\mathrm{K}^{+}$channel activity (Fig. 4, panel C). 

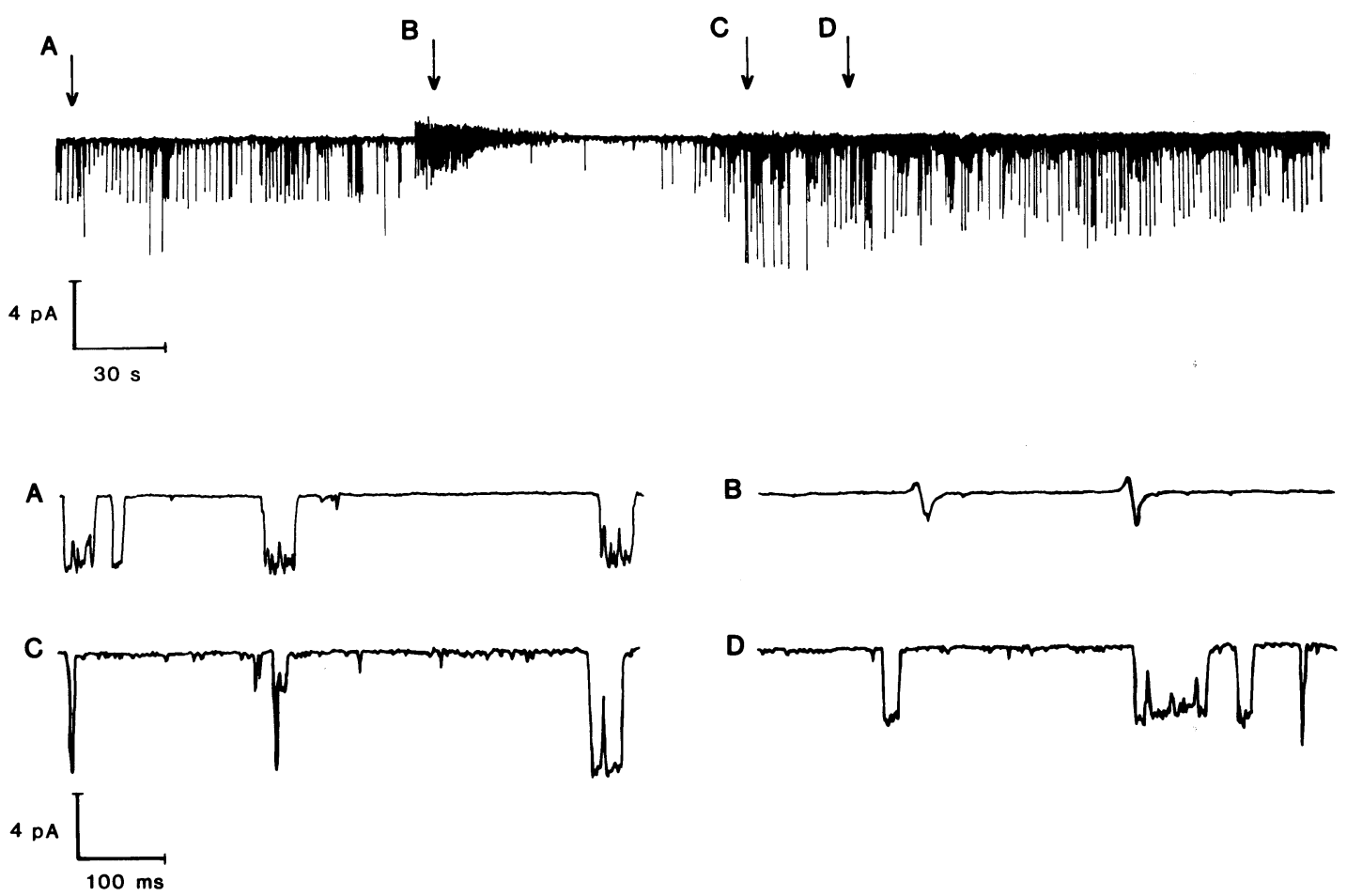

Fig. 3. Effects of $10 \mu \mathrm{g} / \mathrm{ml}$ rhodamine $\mathrm{B}$ on the channel activity of a mouse $\beta$-cell in the presence of $3 \mathrm{mM}$ glucose studied by the patch clamp technique with HEPES buffered media ( $\mathrm{pH}$ 7.4). The extracellular medium contained (mM): $\mathrm{NaCl} \mathrm{138,} \mathrm{KCl} 5.6, \mathrm{CaCl}_{2} 2.6, \mathrm{MgCl}_{2} 1.2 \mathrm{When}$ the medium was included in a patch clamp pipette $\mathrm{NaCl}$ was replaced with equimolar $\mathrm{KCl}$. The top trace shows an abrupt stop in the ATP-regulated $\mathrm{K}^{+}$channel activity and the onset of action potentials shortly after the introduction of rhodamine. Prolonged exposure resulted in the disappearance of the action potentials and in a gradual increase of channel activity. The lower four traces show sections (denoted A-D) of the top trace with a greater time resolution. A larger channel type is apparent in traces C-D.

\section{DISCUSSION}

Most of the procedures described for rapid changes of extracellular medium are intended for the superfusion of membrane fragments studied by the patch clamp technique $(1,2,11,13,16)$. Rapid medium changes around intact cells encounter difficulties in that these are more sensitive to mechanical stress and high flow rates than are membrane fragments. In the insideout configuration of the patch clamp technique, the membrane can even withstand the passage of an air bubble used to separate media $(1,2,11,13,16)$. Moreover, the excised membrane fragments can easily be positioned relative to a fixed superfusion device with the aid of the measuring pipette $(1,2,11,13,16)$. The present procedure for changing media around individual cells is a development of the technique originally proposed by Krishtal and Pidoplichko (12). An advantage of the Utube approach compared with many other systems used for changing media is that the device can easily be moved relative to a fixed cell.
Using the U-tube technique, Fenwick et al. (5) made complete medium changes $300 \mu \mathrm{m}$ from the ejection hole in $200 \mathrm{~ms}$. After exposure for $10 \mathrm{~s}$, medium was sucked back reversing the change with a half time of $5 \mathrm{~s}$. The perfusion of the U-tube can be achieved in two ways. Either well defined pressures can be applied to the arms of the U-tube to obtain constant flows (pressure control), or the flow rates can be controlled independently of pressure (rate control), for example by using a syringe pump. It is important that neither of the two flows connected to the arms of the U-tube changes its rate independently of the other, except when switching the valve. Such stability can be difficult to achieve at low flow rates in a pressure controlled system. To meet the difficulties of pressure control we have chosen to use a purpose-made syringe pump. For maintaining the flow pattern the pistons were connected, forcing the syringes to dispense and suck fluid in a coupled manner. In having a compact design and being practically free of vibrations, this syringe pump could be placed near the measuring equipment. Electromagnetic noise in patch 


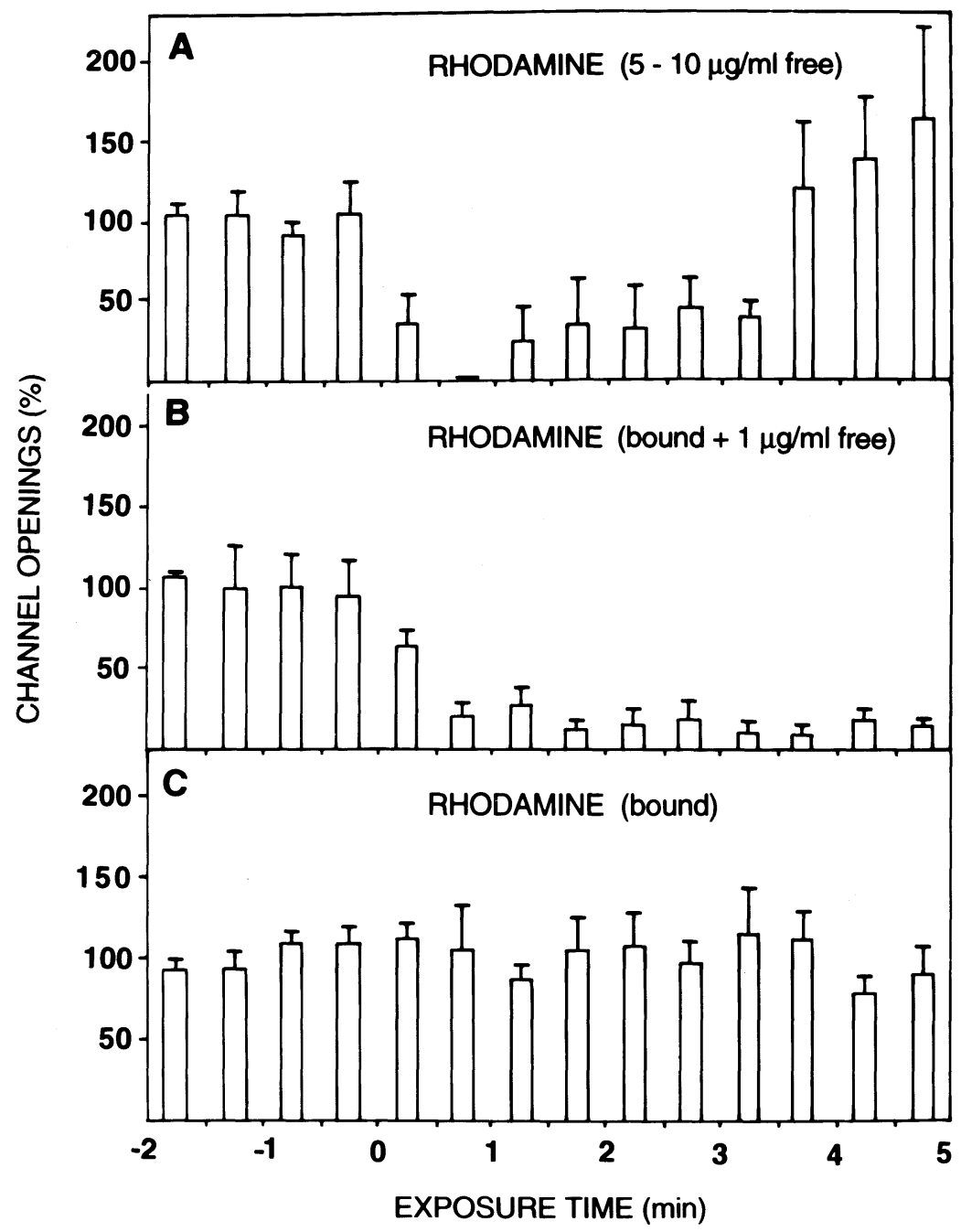

Fig. 4. Effects of rhodamine $\mathrm{B}$ on ATP-regulated $\mathrm{K}^{+}$channel activity in mouse $\beta$-cells in the presence of 3 mM glucose. The measurements were carried out as in Fig. 3. At time zero the extracellular medium was supplemented with $5-10 \mu \mathrm{g} / \mathrm{ml}$ free rhodamine (panel A) or $10 \mu \mathrm{g} / \mathrm{ml}$ rhodamine bound to dextran either in the presence (panel B) or in the absence (panel C) of $1 \mu \mathrm{g} / \mathrm{ml}$ free rhodamine. Channel activities are expressed as percentages of the average number of openings measured during 30 second periods before adding rhodamine $B$. Mean values \pm SEM for 4-6 experiments.

clamp studies was minimized by placing the remote driving unit for the syringe pump outside the Faraday cage that accomodated the rest of the equipment.

The U-tube described was not only smaller than those previously employed, but it was also made from plastic instead of fragile glass. The U-tube was held with its ejection hole only $50 \mu \mathrm{m}$ from the cell, minimizing the time for the medium to reach it. The premise for obtaining rapid medium changes at a flow rate of $1 \mu \mathrm{l} / \mathrm{min}$ was the presence of a sharp boundary between the media. It was found that recoil pulses caused by switching the valve slowed down the time for reaching final concentrations by perturbing the boundary. The pressure transients also mechanically disturbed the cells, as was evident from visual inspection and changes in electrical properties. The problem was solved by letting the pressure transients compress an air bubble rather than allow them to be transmitted along the narrow tubing to the ejection hole. The damping was controlled by the volume of the bubble. In this way it was possible to make rapid medium changes without detectable disturbances to the cell.

An advantage of a flow rate as low as $1 \mu \mathrm{l} / \mathrm{min}$ was that it enabled the deposition of medium in a restricted area. In this way it was possible to create a well defined microenvironment surrounding an individual cell. For the successful application of the U-tube technique it was necessary to be able to inspect visually the changes of medium by adding an indicator. Methylene blue was used as such an indicator in the introductory model 
studies, but it had to be discontinued in view of its adverse effects on living cells $(6,15)$. In the search for more suitable indicators, fluorescent substances were of particular interest in requiring only low concentrations for detection. Possible effects of such indicators were tested on pancreatic $\beta$-cells with regard to their influence on metabolism as indicated by the activity of the ATP-regulated $\mathrm{K}^{+}$channels.

To minimize photodamage to the cells, it is preferable to use fluorescent indicators, which are excitable at visible wavelengths. Testing the widely used indicator rhodamine $\mathrm{B}$, it was found that this stain in itself had effects of a complex nature. When added at a concentration of $5-10 \mu \mathrm{g} / \mathrm{ml}$, rhodamine B initially suppressed the activity of the ATP-regulated $\mathrm{K}^{+}$channels with the resulting appearance of action potentials. This was followed by a gradual increase of the $\mathrm{K}^{+}$channel activity, probably reflecting an impaired ATP production. It was not unexpected to observe indications of a disturbed ATP production in the presence of high concentrations of the fluorescent indicator, since the related compound rhodamine 123 is used as a mitochondrial stain (3). When administering the fluorescent stain at a concentration of $1 \mu \mathrm{g} / \mathrm{ml}$, which is below the limit for visual inspection, the initial suppression of channel activity remained during the whole experiment. It will be a matter for further studies to decide whether this suppression reflects a direct rhodamine interaction with the channel protein as seen with hypoglycemic sulfonylureas (17). To obviate side effects of rhodamine B as a marker when locally changing the medium around single cells, it was used covalently bound to dextran. It can be concluded that the improved U-tube technique offers considerable advantages in enabling well controlled medium changes when the substance to be tested is added together with dextran-linked rhodamine B.

Acknowledgements. The authors are indebted to Drs. Nigel Marsden and Avi Ring for helpful advice and for constructive discussions. This study was supported by grants from the Swedish Medical Research Council (12X-562), the Swedish Diabetes Association, the Nordic Insulin Foundation, Novo Nordisk A/S and the Family Ernfors Foundation.

\section{REFERENCES}

1. Akaike, N., Inoue, M., and Krishtal, O.A. 1986. "Concentration clamp" study of gamma-aminobutyric-acid-induced chloride current kinetics in frog sensory neurones. J. Physiol., 379: 171-185.
2. Brett, R.S., Dilger, J.P., Adams, P.R., and Lancaster, B. 1986. A method for the rapid exchange of solutions bathing excised membrane patches. Biophys. J., 50: 987-992.

3. CHEN, L.B. 1989. Fluorescent labeling of mitochondria. In Methods in Cell Biology vol. 29 (Y-L. Wang, and D. Lansing Taylor, eds.). Academic Press, San Diego, pp 103-123.

4. Cobbold, P.H. and Rink, T.J. 1987. Fluorescence and bioluminiscence measurement of cytoplasmic free calcium. Biochem. J., 248: 313-328.

5. Fenwick, E.M., Marty, A., and Neher, E. 1982. A patchclamp study of bovine chromaffin cells and of their sensitivity to acetylcholine. J. Physiol., 331: 577-597.

6. Grapengiesser, E. 1993. Cell photodamage, a potential hazard when measuring cytoplasmic $\mathrm{Ca}^{2+}$ with fura-2. Cell Struct. Funct., 18: 13-17.

7. Grapengiesser, E., Berts, A., Saha, S., Lund, P-E., Gylfe, E., and Hellman, B. 1993. Dual effects of $\mathrm{Na} / \mathrm{K}$ pump inhibition on cytoplasmic $\mathrm{Ca}^{2+}$ oscillations in pancreatic $\beta$-cells. Arch. Biochem. Biophys., 300: 372-377.

8. Grynkiewicz, G., Poennie, M., and Tsien, R.Y. 1985. A new generation of $\mathrm{Ca}^{2+}$ indicators with greatly improved fluorescence properties. J. Biol. Chem., 260: 3440-3450.

9. Hamill, O.P., Marty, A., Neher, E., Sakmann, B., and SigWORTH, F.J. 1981. Improved patch-clamp techniques for high resolution current recording from cells and cell-free membrane patches. Pflügers Arch., 391: 85-100.

10. Harootunian, A.T., Kao, J.P.Y., Eckert, B.K., and Tsien, R.Y. 1989. Fluorescence ratio imaging of cytosolic free $\mathrm{Na}^{+}$ in individual fibroblasts and lymphocytes. J. Biol. Chem., 264: 19458-19467.

11. KaKei, M. and Ashcroft, F.M. 1987. A microflow superfusion system for use with excised patches. Pflügers Arch., 409: 337-341.

12. Krishtal, O.A. and PidoplichKo, V.I. 1980. A receptor for protons in the nerve cell membrane. Neuroscience, 5: 23252327.

13. Maconochie, D.J. and Knight, D.E. 1989. A method for making solution changes in the sub-millisecond range at the tip of a patch pipette. Pflügers Arch., 414: 589-596.

14. Mayer, B., Brunner, F., and Schmidt, K. 1993. Inhibition of nitric oxide synthesis by methylene blue. Biochem. Pharmacol., 45: 367-374.

15. Schrader, M.C., Simplaceanu, V., and Ho, C. 1993. Measurement of fluxes through the pentose phosphate pathway in erythrocytes from individuals with sickle cell anemia by carbon13 nuclear magnetic resonance spectroscopy. Biochim. Biophys. Acta, 1182: 179-188.

16. Yellen, G. 1982. Single $\mathrm{Ca}^{2+}$-activated non-selective cation channel in neuroblastoma. Nature, 296: 357-359.

17. Panten, U., Heipel, C., Rosenberger, F., Scheffer, K., ZünKleR, B.J., and SchWANSTECher, C. 1990. Tolbutamidesensitivity of the adenosine $5^{\prime}$-triphosphate-dependent $\mathrm{K}^{+}$channel in mouse pancreatic B-cells. Naunyn-Schmiedeberg's Arch. Parmacol., 342: 566-574.

(Received for publication, August 9, 1994 and in revised form, September 16, 1994) 